\title{
On the controllability of fractional functional integro-differential systems with an infinite delay in Banach spaces
}

\author{
Chokkalingam Ravichandran ${ }^{1}$ and Dumitru Baleanu ${ }^{2,3,4 *}$
}

\author{
"Correspondence: \\ dumitru@cankaya.edu.tr \\ ${ }^{2}$ Department of Mathematics and \\ Computer Science, Faculty of Arts \\ and Sciences, Cankaya University, \\ Ankara, 06530, Turkey \\ ${ }^{3}$ Department of Chemical and \\ Materials Engineering, Faculty of \\ Engineering, King Abdulaziz \\ University, Jeddah, Saudi Arabia \\ Full list of author information is \\ available at the end of the article
}

\begin{abstract}
In this manuscript, we study the sufficient conditions for controllability for fractional functional integro-differential systems involving the Caputo fractional derivative of order $\alpha \in(0,1]$ in Banach spaces. Our main approach is based on fractional calculus, the properties of characteristic solution operators, Mönch's fixed point theorem via measures of noncompactness. Particularly, these results are under some weakly compactness conditions. An example is presented in the end to show the applications of the obtained abstract results.
\end{abstract}

MSC: $26 \mathrm{~A} 33 ; 93 \mathrm{~B} 05 ; 47 \mathrm{H} 08 ; 47 \mathrm{H} 10$

Keywords: controllability; Caputo fractional derivative; measures of noncompactness; fixed point theorem

\section{Introduction}

The theory of fractional differential and integral equations have been proved to be valuable tools and effective in the modeling of many phenomena in various fields of engineering and scientific disciplines such as physics, chemistry, biology, control theory, signal and image processing, biophysics, blood flow phenomena, aerodynamics and so on. For more details on fractional calculus theory, one can see the monographs of Kilbas et al. [1], Lakshmikantham et al. [2], Miller and Ross [3] and Podlubny [4], Baleanu et al. [5], Tarasov [6], and the papers [7-15] as well as the references therein.

As it is known, the concept of controllability is an important property (quantitative and qualitative) of a control system, and the controllability property plays a crucial role in many control problems and both finite and infinite-dimensional spaces. Recently, the controllability for a fractional dynamical system has become a very active area to this field. Controllability of linear systems in finite dimensional spaces is well established in [16] and controllability of fractional evolution dynamical systems in a finite dimensional space is discussed, for example, in $[7,8,14,17]$.

Very recently, the authors Wang and Zhou [18] found some conditions guaranteeing the complete controllability of fractional evolution systems without assuming the compactness of characteristic solution operators by means of the Mönch fixed point technique and the measures of noncompactness. Wang et al. [19] established two sufficient conditions for nonlocal controllability for fractional evolution systems. These theorems guarantee the effectiveness of controllability results under some weakly noncompactness conditions. The

o2013 Ravichandran and Baleanu; licensee Springer. This is an Open Access article distributed under the terms of the Creative Commons Attribution License (http://creativecommons.org/licenses/by/2.0), which permits unrestricted use, distribution, and reproduction in any medium, provided the original work is properly cited. 
authors Ji et al. [20] derived the nonlocal controllability of an impulsive differential evolution system and the evolution part is not compact by means of Mönch's fixed point theorem via measures of noncompactness. Many authors investigated the controllability problem, there are different methods and techniques for various types of nonlinear systems and fixed point theorems; the details of these papers can be found in [11,13-15, 21-26] and the references therein. From the above literature survey, to our knowledge, controllability of fractional functional integro-differential systems with infinite delay by using Mönch's fixed point theorem via MNC properties and an abstract phase space have not been studied fully. Motivated by this fact and the articles [18-20], in this manuscript we are concerned with the controllability of fractional integro-differential systems with infinite delay of the form

$$
\begin{aligned}
& { }^{C} D^{q} x(t)=A x(t)+f\left(t, x_{t}, \int_{0}^{t} h\left(t, s, x_{s}\right) d s\right)+B u(t), \quad t \in J:=[0, b], \\
& x_{0}=\phi \in \mathcal{B}_{h}, \quad t \in(-\infty, 0]
\end{aligned}
$$

where ${ }^{C} D^{q}$ is the Caputo fractional derivative of order $0<q<1, A$ is the infinitesimal generator of a strongly continuous semigroup $\{T(t), t \geq 0\}$ in a Banach space $X$, which means that there exists $M_{1} \geq 1$ such that $\sup _{t \in J}\|T(t)\| \leq M_{1}, f: J \times \mathcal{B}_{h} \times X, h: J \times J \times \mathcal{B}_{h}$ are given functions, where $\mathcal{B}_{h}$ is a phase space to be defined later. The control function $u(\cdot)$ is given $L^{2}(J, U)$, a Banach space of admissible control functions, with $U$ as a Banach space. Finally, $B$ is a bounded linear operator from $U$ into $X$. The histories $x_{t}:(-\infty, 0] \rightarrow X$, defined by $x_{t}(s)=x(t+s), s \leq 0$, belong to some abstract phase space $\mathcal{B}_{h}$.

The rest of this manuscript is organized as follows. In Section 2 , we briefly present some basic notations and preliminaries. In Section 3, we establish some sufficient conditions for controllability of fractional integro-differential evolution systems with infinite delay. Finally, an example is given to illustrate the results reported in Section 4.

\section{Preliminaries}

In this section, we mention notations, definitions, lemmas and preliminary facts needed to establish our main results.

Throughout this paper, we denote by $X$ a Banach space with the norm $\|\cdot\|$. Let $Y$ be another Banach space, let $L_{b}(X, Y)$ denote the space of bounded linear operators from $X$ to $Y$. We also use $\|f\|_{L^{p}\left(, R^{+}\right)}$norm of $f$ whenever $f \in L^{p}\left(J, R^{+}\right)$for some $p$ with $1 \leq p \leq \infty$. Let $L^{p}(J, X)$ denote the Banach space of functions $f: J \times \mathcal{B}_{h} \times X \rightarrow X$ which are Bochner integrable normed by $\|f\|_{L^{p}(J, X)}$. Let $\mathcal{C}(J, X)$ be the Banach space of continuous functions from $J$ into $X$ with the usual supremum norm $\|x\|_{\mathcal{C}}:=\sup _{t \in J}\|x(t)\|$ for $x \in \mathcal{C}$.

Now we define the abstract phase space $\mathcal{B}_{h}$, which was used in [27]. Assume that $h$ : $(-\infty, 0] \rightarrow(0,+\infty)$ is a continuous function with $l=\int_{-\infty}^{0} h(t) d t<+\infty$. For any $a>0$, we define

$$
\mathcal{B}=\{\psi:[-a, 0] \rightarrow X \text { such that } \psi(t) \text { is bounded and measurable }\}
$$

and equip the space $\mathcal{B}$ with the norm

$$
\|\psi\|_{[-a, 0]}=\sup _{s \in[-a, 0]}|\psi(s)|, \quad \forall \psi \in \mathcal{B} .
$$


Let us define

$$
\begin{aligned}
\mathcal{B}_{h}= & \left\{\psi:(-\infty, 0] \rightarrow X \text { such that for any } c>0,\left.\psi\right|_{[-c, 0]} \in \mathcal{B}\right. \\
& \text { and } \left.\int_{-\infty}^{0} h(s)\|\psi\|_{[s, 0]} d s<+\infty\right\} .
\end{aligned}
$$

If $\mathcal{B}_{h}$ is endowed with the norm

$$
\|\psi\|_{\mathcal{B}_{h}}=\int_{-\infty}^{0} h(s)\|\psi\|_{[s, 0]} d s, \quad \forall \psi \in \mathcal{B}_{h}
$$

then it is clear that $\left(\mathcal{B}_{h},\|\cdot\|_{\mathcal{B}_{h}}\right)$ is a Banach space.

Now we consider the space

$$
\mathcal{B}_{h}^{\prime}=\left\{x:(-\infty, b] \rightarrow X \text { such that }\left.x\right|_{J} \in C(J, X), x_{0}=\phi \in \mathcal{B}_{h}\right\} .
$$

Set $\|\cdot\|_{b}$ to be a seminorm in $\mathcal{B}_{h}^{\prime}$ defined by

$$
\|x\|_{b}=\|\phi\|_{\mathcal{B}_{h}}+\sup \{|x(s)|: s \in[0, b]\}, \quad x \in \mathcal{B}_{h}^{\prime} .
$$

Lemma $2.1([27,28])$ Assume $x \in \mathcal{B}_{h}^{\prime}$, then for $t \in J, x_{t} \in \mathcal{B}_{h}$. Moreover,

$$
l|x(t)| \leq\left\|x_{t}\right\|_{\mathcal{B}_{h}} \leq\|\phi\|_{\mathcal{B}_{h}}+l \sup _{s \in[0, t]}|x(s)|,
$$

where $l=\int_{-\infty}^{0} h(t) d t<+\infty$.

Let us recall the following known definitions. For more details, see $[1,5]$ and the references therein.

Definition 2.1 The fractional integral of order $\alpha$ with the lower limit zero for a function $f$ is defined as

$$
I^{\alpha} f(t)=\frac{1}{\Gamma(\alpha)} \int_{0}^{t} \frac{f(s)}{(t-s)^{1-\alpha}} d s, \quad t>0, \alpha>0
$$

provided the right-hand side is point-wise defined on $[0, \infty)$, where $\Gamma(\cdot)$ is the gamma function, which is defined by $\Gamma(\alpha)=\int_{0}^{\infty} t^{\alpha-1} e^{-t} d t$.

Definition 2.2 The Riemann-Liouville fractional derivative of order $\alpha>0, n-1<\alpha<n$, $n \in N$, is defined as

$$
{ }^{(R-L)} D_{0+}^{\alpha} f(t)=\frac{1}{\Gamma(n-\alpha)}\left(\frac{d}{d t}\right)^{n} \int_{0}^{t}(t-s)^{n-\alpha-1} f(s) d s,
$$

where the function $f(t)$ has absolutely continuous derivative up to order $(n-1)$. 
Definition 2.3 The Caputo derivative of order $\alpha$ for a function $f:[0, \infty) \rightarrow R$ can be written as

$$
D^{\alpha} f(t)=D^{\alpha}\left(f(t)-\sum_{k=0}^{n-1} \frac{t^{k}}{k !} f^{(k)}(0)\right), \quad t>0, n-1<\alpha<n .
$$

Remark 2.1 (i) If $f(t) \in C^{n}[0, \infty)$, then

$$
{ }^{C} D^{\alpha} f(t)=\frac{1}{\Gamma(n-\alpha)} \int_{0}^{t} \frac{f^{(n)}(s)}{(t-s)^{\alpha+1-n}} d s=I^{n-\alpha} f^{(n)}(t), \quad t>0, n-1<\alpha<n .
$$

(ii) The Caputo derivative of a constant is equal to zero.

(iii) If $f$ is an abstract function with values in $X$, then integrals which appear in Definitions 2.1 and 2.2 are taken in Bochner's sense.

Definition 2.4 ([18, 29]) A continuous function $x:(-\infty, b] \rightarrow X$ is said to be a mild solution of system (1.1)-(1.2) if $x_{0}=\phi \in \mathcal{B}_{h}$ on $(-\infty, 0]$ and the following integral equation

$$
\begin{aligned}
x(t)= & \mathcal{T}(t) \phi(0)+\int_{0}^{t}(t-s)^{q-1} \mathcal{S}(t-s)\left[f\left(s, x_{s}, \int_{0}^{s} h\left(s, \tau, x_{\tau}\right) d \tau\right)\right. \\
& +B u(s)] d s, \quad t \in J,
\end{aligned}
$$

is satisfied, where $\mathcal{T}(\cdot)$ and $\mathcal{S}(\cdot)$ are called characteristic solution operators and given by

$$
\mathcal{T}=\int_{0}^{\infty} \xi_{q}(\theta) T\left(t^{q} \theta\right) d \theta, \quad \mathcal{S}=q \int_{0}^{\infty} \theta \xi_{q}(\theta) T\left(t^{q} \theta\right) d \theta
$$

and for $\theta \in(0, \infty)$

$$
\begin{aligned}
& \xi_{q}(\theta)=\frac{1}{q} \theta^{-1-\frac{1}{q}} \bar{w}_{q}\left(\theta^{-\frac{1}{q}}\right) \geq 0, \\
& \bar{w}_{q}(\theta)=\frac{1}{\pi} \sum_{n=1}^{\infty}(-1)^{n-1} \theta^{-n q-1} \frac{\Gamma(n q+1)}{n !} \sin (n \pi q) .
\end{aligned}
$$

Here, $\xi_{q}$ is a probability density function defined on $(0, \infty)$, that is,

$$
\xi_{q}(\theta) \geq 0, \quad \theta \in(0, \infty) \text { and } \quad \int_{0}^{\infty} \xi_{q}(\theta) d \theta=1
$$

The following results of $\mathcal{T}(\cdot)$ and $\mathcal{S}(\cdot)$ will be used throughout this paper.

Lemma 2.2 ([19]) The operators $\mathcal{T}$ and $\mathcal{S}$ have the following properties:

(i) For any fixed $t \geq 0, \mathcal{T}$ and $\mathcal{S}$ are linear and bounded operators, that is, for any $x \in X$,

$$
\|\mathcal{T}(t) x\| \leq M\|x\| \quad \text { and } \quad\|\mathcal{S}(t) x\| \leq \frac{q M}{\Gamma(1+q)}\|x\| .
$$

(ii) $\{\mathcal{T}(t), t \geq 0\}$ and $\{\mathcal{S}(t), t \geq 0\}$ are strongly continuous. 
(iii) For $t \in J$ and any bounded subsets $D \subset X, t \rightarrow\{\mathcal{T}(t) x: x \in D\}$ and $t \rightarrow\{\mathcal{S}(t) x: x \in D\}$ are equicontinuous if $\left\|T\left(t_{2}^{q}(\theta)\right) x-T\left(t_{1}^{q}(\theta)\right) x\right\| \rightarrow 0$ with respect to $x \in D$ as $t_{2} \rightarrow t_{1}$ for each fixed $\theta \in[0, \infty]$.

Definition 2.5 System (1.1) with (1.2) is said to be controllable on the interval $J$ if for every continuous initial function $\phi \in \mathcal{B}_{h}, x^{1} \in X$, there exists a control $u \in L^{2}(J, U)$ such that the mild solution $x(t)$ of (1.1) with (1.2) satisfies $x(b)=x^{1}$.

Moreover, let us recall some definitions and properties of the measures of noncompactness.

Definition 2.6 ([20]) Let $E^{+}$be the positive cone of an ordered Banach space $(E, \leq)$. A function $\Phi$ defined on the set of all bounded subsets of the Banach space $X$ with values in $E^{+}$is called a measure of noncompactness (MNC) on $X$ iff $\Phi(\overline{c o} \Omega)=\Phi(\Omega)$ for all bounded subsets $\Omega \subseteq X$, where $\overline{c o} \Omega$ stands for the closed convex hull of $\Omega$.

The MNC $\Phi$ is said to be:

(1) monotone iff for all bounded subsets $\Omega_{1}, \Omega_{2}$ of $X$, we have

$$
\left(\Omega_{1} \subseteq \Omega_{2}\right) \quad \Rightarrow \quad\left(\Phi\left(\Omega_{1}\right) \leq \Phi\left(\Omega_{2}\right)\right)
$$

(2) nonsingular iff $\Phi(\{a\} \cup \Omega)=\Phi(\Omega)$ for every $a \in X, \Omega \subset X$;

(3) regular iff $\Phi(\Omega)=0$ if and only if $\Omega$ is relatively compact in $X$.

One of the many examples of MNC is the noncompactness measure of Hausdorff $\beta$ defined on each bounded subset $\Omega$ of $X$ by

$$
\begin{aligned}
\beta(\Omega)= & \inf \{\epsilon>0 ; \Omega \text { can be covered by a finite } \\
& \text { number of balls of radii smaller than } \epsilon\} .
\end{aligned}
$$

It is well known that MNC $\beta$ enjoys the above properties and other properties (see [30, 31]): For all bounded subsets $\Omega, \Omega_{1}, \Omega_{2}$ of $X$,

(4) $\beta\left(\Omega_{1}+\Omega_{2}\right) \leq \beta\left(\Omega_{1}\right)+\beta\left(\Omega_{2}\right)$, where $\Omega_{1}+\Omega_{2}=\left\{x+y: x \in \Omega_{1}, y \in \Omega_{2}\right\}$;

(5) $\beta\left(\Omega_{1} \cup \Omega_{2}\right) \leq \max \left\{\beta\left(\Omega_{1}\right), \beta\left(\Omega_{2}\right)\right\}$;

(6) $\beta(\lambda \Omega) \leq|\lambda| \beta(\Omega)$ for any $\lambda \in R$;

(7) If the map $Q: D(Q) \subseteq X \rightarrow Z$ is Lipschitz continuous with constant $k$, then $\beta_{Z}(Q \Omega) \leq k \beta(\Omega)$ for any bounded subset $\Omega \subseteq D(Q)$, where $Z$ is a Banach space.

Lemma 2.3 ([30]) If $W \subset C([a, b], X)$ is bounded and equicontinuous, then $\beta(W(t))$ is continuous for $t \in[a, b]$ and

$$
\beta(W)=\sup \{\beta(W(t)), t \in[a, b]\}, \quad \text { where } W(t)=\{x(t): x \in W\} \subseteq X .
$$

Theorem 2.1 ([19, 32]) If $\left\{u_{n}\right\}_{n=1}^{\infty}$ is a sequence of Bochner integrable functions from $J$ into $X$ with the estimation $\left\|u_{n}(t)\right\| \leq \mu(t)$ for almost all $t \in J$ and every $n \geq 1$, where $\mu \in L^{1}(J, R)$, then the function $\psi(t)=\beta\left(\left\{u_{n}(t): n \geq 1\right\}\right)$ belongs to $L^{1}(J, R)$ and satisfies $\beta\left(\left\{\int_{0}^{t} \psi(s) d s: n \geq\right.\right.$ $1\}) \leq 2 \int_{0}^{t} \psi(s) d s$. 
The following fixed point theorem, a nonlinear alternative of Mönch's type, plays a key role in our proof of system (1.1)-(1.2).

Lemma 2.4 ([33, Theorem 2.2]) Let D be a closed convex subset of a Banach space $X$ and $0 \in D$. Assume that $F: D \rightarrow X$ is a continuous map which satisfies Monch's condition, that is, $(M \subseteq D$ is countable, $M \subseteq \overline{c o}(\{0\} \cup F(M)) \Rightarrow \bar{M}$ is compact $)$. Then $F$ has a fixed point in $D$.

\section{Controllability results}

In this section, we establish the controllability results for problem (1.1)-(1.2). In order to prove the main theorem of this section, we list the following hypotheses.

$\left(\mathrm{H}_{1}\right) \quad$ (i) $A$ generates a strongly continuous semigroup $\{T(t): t \geq 0\}$ in $X$.

(ii) For all bounded subsets $D \subset X$ and $x \in D,\left\|T\left(t_{2}^{q} \theta\right) x-T\left(t_{1}^{q} \theta\right) x\right\| \rightarrow 0$ as $t_{2} \rightarrow t_{1}$ for each fixed $\theta \in(0, \infty)$.

$\left(\mathrm{H}_{2}\right)$ The function $f: J \times \mathcal{B}_{h} \times X \rightarrow X$ satisfies:

(i) $f(\cdot, \phi, x)$ is measurable for all $(\phi, x) \in \mathcal{B}_{h} \times X$ and $f(t, \cdot, \cdot)$ is continuous for a.e. $t \in J$.

(ii) There exist a constant $q_{1} \in(0, q)$ and $m \in L^{\frac{1}{q_{1}}}\left(J, R^{+}\right)$and a nondecreasing continuous function $\Omega: R^{+} \rightarrow R^{+}$such that $\|f(t, \phi, x)\| \leq m(t) \Omega\left(\|\phi\|_{\mathcal{B}_{h}}+\|x\|\right)$, for all $(t, \phi, x) \in J \times \mathcal{B}_{h} \times X$, where $\Omega$ satisfies $\liminf _{n \rightarrow \infty} \frac{\Omega(n)}{n}=0$.

(iii) There exist a constant $q_{2} \in(0, q)$ and $h \in L^{\frac{1}{q_{2}}}\left(J, R^{+}\right)$such that, for any bounded subset $D_{1} \subset X, F_{1} \subset \mathcal{B}_{h}$,

$$
\beta\left(f\left(t, F_{1}, D_{1}\right)\right) \leq \eta(t)\left[\sup _{-\infty<\theta \leq 0} \beta\left(F_{1}(\theta)\right)+\beta\left(D_{1}\right)\right] \text { for a.e. } t \in J
$$

where $\beta$ is the Hausdorff MNC.

$\left(\mathrm{H}_{3}\right)$ The function $h: J \times \mathcal{B}_{h} \times X \rightarrow X$ satisfies:

(i) $h(\cdot, \phi, x)$ is measurable for $(\phi, x) \in \mathcal{B}_{h} \times X$ and $h(t, \cdot, \cdot)$ is continuous for a.e. $t \in J$.

(ii) There exists a constant $H_{0}>0$ such that $\|h(t, s, \phi)\| \leq H_{0}\left(1+\|\phi\|_{\mathcal{B}_{h}}\right)$ for all $\phi \in \mathcal{B}_{h}$.

(iii) There exists $\zeta \in L^{1}\left(J^{2}, R^{+}\right)$such that for any bounded subset $D_{2} \subset X$,

$$
\beta\left(h\left(t, s, D_{2}\right)\right) \leq \zeta(t, s)\left[\sup _{-\infty<\theta \leq 0} \beta\left(D_{2}(\theta)\right)\right] \quad \text { for a.e. } t \in J
$$

with $\zeta^{*}=\sup _{s \in J} \int_{0}^{s} \zeta(t, \tau) d \tau<\infty$.

$\left(\mathrm{H}_{4}\right) \quad$ (i) The linear operator $B: L^{2}(J, U) \rightarrow L^{1}(J, X)$ is bounded, $W: L^{2}(J, U) \rightarrow X$ defined by $W u=\int_{0}^{b}(b-s)^{q-1} \mathcal{S}(t-s) B u(s) d s$ has an inverse operator $W^{-1}$ which takes values in $L^{2}(J, U) / \operatorname{Ker} W$ and there exist two constants $M_{2}, M_{3}>0$ such that $\|B\| \leq M_{2}$ and $\left\|W^{-1}\right\| \leq M_{3}$. (For the construction of the operator $W$ and its inverse, see [34].)

(ii) There exist a constant $q_{0} \in(0, q)$ and $K_{W} \in L^{\frac{1}{q_{0}}}\left(J, R^{+}\right)$such that for any bounded subset $Q \subset X, \beta\left(\left(W^{-1} Q\right)(t)\right) \leq K_{W}(t) \beta(Q)$. 
For our convenience, let us take

$$
\begin{aligned}
& \kappa_{i}:=\left[\left(\frac{1-q_{i}}{q-q_{i}}\right) b^{\frac{q-q_{i}}{1-q_{i}}}\right]^{1-q_{i}}, \quad i=0,1,2 ; \\
& M_{4}:=\kappa_{1}\|m\|_{L^{\frac{1}{q_{1}}}\left(J, R^{+}\right)}, \quad M_{5}:=\kappa_{0}\left\|K_{W}\right\|_{L^{\frac{1}{q_{0}}}\left(J, R^{+}\right)}
\end{aligned}
$$

and $M_{6}=\kappa_{2}\|\eta\|_{L^{\frac{1}{q_{2}}}\left(J, R^{+}\right)}$.

Theorem 3.1 Assume that hypotheses $\left(\mathrm{H}_{1}\right)-\left(\mathrm{H}_{4}\right)$ are satisfied. Then system (1.1)-(1.2) is controllable on J, provided that

$$
L^{*}=\left[1+\frac{2 M_{1} M_{2} M_{5} q}{\Gamma(1+q)}\right]\left(1+2 \zeta^{*}\right) \frac{2 M_{1} M_{6} q}{\Gamma(1+q)}<1 \quad \text { for some } \frac{1}{2}<q<1 .
$$

Proof Using hypothesis $\left(\mathrm{H}_{4}\right)$, for an arbitrary function $x(\cdot) \in \mathcal{C}$, we define the control $u_{x}(t)$ by

$$
\begin{aligned}
u_{x}(t)= & W^{-1}\left[x^{1}-\mathcal{T}(b) \phi(0)\right. \\
& \left.-\int_{0}^{b}(b-s)^{q-1} \mathcal{S}(b-s) f\left(s, x_{s}, \int_{0}^{s} h\left(s, \tau, x_{\tau}\right) d \tau\right) d s\right](t), \quad t \in J .
\end{aligned}
$$

We show that, using this control, the operator $\Phi: \mathcal{B}_{h}^{\prime} \rightarrow \mathcal{B}_{h}^{\prime}$ defined by

$$
\Phi x(t)= \begin{cases}\phi(t), & t \in(-\infty, 0], \\ \mathcal{T}(t) \phi(0)+\int_{0}^{t}(t-s)^{q-1} \mathcal{S}(t-s)\left\{f\left(s, x_{s}, \int_{0}^{s} h\left(s, \tau, x_{\tau}\right) d \tau\right)\right. & \\ \left.\quad+B u_{x}(s)\right\} d s, & t \in J,\end{cases}
$$

has a fixed point. This fixed point is then a solution of a given system. Clearly, $\Phi x(b)=x^{1}$, which implies the fractional system (1.1)-(1.2) is controllable on $J$.

For $\phi \in \mathcal{B}_{h}$, we define $\hat{\phi}$ by

$$
\hat{\phi}(t)= \begin{cases}\phi(t), & t \in(-\infty, 0] \\ \mathcal{T}(t) \phi(0), & t \in J\end{cases}
$$

then $\hat{\phi} \in \mathcal{B}_{h}^{\prime}$. Let $x(t)=y(t)+\hat{\phi}(t),-\infty<t \leq b$. It is easy to see that $x$ satisfies (2.1) if and only if $y$ satisfies $y_{0}=0$ and

$$
y(t)=\int_{0}^{t}(t-s)^{q-1} \mathcal{S}(t-s)\left[f\left(s, y_{s}+\hat{\phi}_{s}, \int_{0}^{s} h\left(s, \tau, y_{\tau}+\hat{\phi}_{\tau}\right) d \tau\right)+B u_{y}(s)\right] d s,
$$

where

$$
\begin{aligned}
u_{y}(s)= & W^{-1}\left[x^{1}-\mathcal{T}(b) \phi(0)\right. \\
& \left.-\int_{0}^{b}(b-s)^{q-1} \mathcal{S}(b-s) f\left(s, y_{s}+\hat{\phi}_{s}, \int_{0}^{s} h\left(s, \tau, y_{\tau}+\hat{\phi}_{\tau}\right) d \tau\right) d s\right](t) .
\end{aligned}
$$


Let $\mathcal{B}_{h}^{\prime \prime}=\left\{y \in \mathcal{B}_{h}^{\prime}: y_{0}=0 \in \mathcal{B}_{h}\right\}$. For any $y \in \mathcal{B}_{h}^{\prime \prime}$,

$$
\begin{aligned}
\|y\|_{b} & =\left\|y_{0}\right\|_{\mathcal{B}_{h}}+\sup \{\|y(s)\|: 0 \leq s \leq b\} \\
& =\sup \{\|y(s)\|: 0 \leq s \leq b\},
\end{aligned}
$$

thus $\left(\mathcal{B}_{h}^{\prime \prime},\|\cdot\|_{b}\right)$ is a Banach space. Set $B_{r}=\left\{y \in \mathcal{B}_{h}^{\prime \prime}:\|y\|_{b} \leq r\right\}$ for some $r>0$, then $B_{r} \subseteq \mathcal{B}_{h}^{\prime \prime}$ is uniformly bounded, and for $y \in B_{r}$, from Lemma 2.1, we have

$$
\begin{aligned}
\left\|y_{t}+\hat{\phi}_{t}\right\|_{\mathcal{B}_{h}} & \leq\left\|y_{t}\right\|_{\mathcal{B}_{h}}+\left\|\hat{\phi}_{t}\right\|_{\mathcal{B}_{h}} \\
& \leq l\left(r+M_{1}|\phi(0)|\right)+\|\phi\|_{\mathcal{B}_{h}}=r^{\prime} .
\end{aligned}
$$

Define the operator $\widetilde{\Phi}: \mathcal{B}_{h}^{\prime \prime} \rightarrow \mathcal{B}_{h}^{\prime \prime}$ by

$$
\widetilde{\Phi} y(t)= \begin{cases}0, & t \in(-\infty, 0], \\ \int_{0}^{t}(t-s)^{q-1} \mathcal{S}(t-s)\left[f\left(s, y_{s}+\hat{\phi}_{s}, \int_{0}^{s} h\left(s, \tau, y_{\tau}+\hat{\phi}_{\tau}\right) d \tau\right)\right. & \\ \left.\quad+B u_{y}(s)\right] d s, & t \in J .\end{cases}
$$

Obviously, the operator $\Phi$ has a fixed point is equivalent to $\widetilde{\Phi}$ has one. So, our main goal is to show that $\widetilde{\Phi}$ has a fixed point and the proof is given in the following steps.

Step 1: There exists $r>0$ such that $\widetilde{\Phi}\left(B_{r}\right) \subseteq B_{r}$.

If this is not true, then for each positive number $r$, there exists a function $y^{r} \in B_{r}$. But $\hat{\Phi}\left(y^{r}\right)$ does not belong to $B_{r}$, i.e., $\left\|\hat{\Phi}\left(y^{r}\right)(t)\right\|>r$ for some $t \in J$.

Then by hypotheses $\left(\mathrm{H}_{2}\right)(\mathrm{iii}),\left(\mathrm{H}_{3}\right)(\mathrm{iii})$ and $\left(\mathrm{H}_{4}\right)(\mathrm{ii})$ and Lemma $2.2(\mathrm{i})$, we have

$$
\begin{aligned}
r< & \left\|\left(\widetilde{\Phi} y^{r}\right)(t)\right\| \\
\leq & \int_{0}^{t}(t-s)^{q-1}\left\|\mathcal{S}(t-s) f\left(s, y_{s}^{r}+\hat{\phi}_{s}, \int_{0}^{s} h\left(s, \tau, y_{\tau}^{r}+\hat{\phi}_{\tau}\right) d \tau\right)\right\| d s \\
& +\int_{0}^{t}(t-s)^{q-1}\left\|\mathcal{S}(t-s) B u_{y^{r}}(s)\right\| d s \\
\leq & \frac{M_{1} M_{4} q}{\Gamma(1+q)} \Omega\left(r^{\prime}+b H_{0}\left(1+r^{\prime}\right)\right)+\frac{M_{1} M_{2} q}{\Gamma(1+q)} \sqrt{\frac{b^{2 q-1}}{2 q-1}}\left\|u_{y^{r}}\right\|_{L^{2}},
\end{aligned}
$$

where

$$
\left\|u_{y^{r}}\right\|_{L^{2}} \leq M_{3}\left[\left\|x_{1}\right\|+M_{1}\|\phi(0)\|+\frac{M_{1} M_{4} q}{\Gamma(1+q)} \Omega\left(r^{\prime}+b H_{0}\left(1+r^{\prime}\right)\right)\right] .
$$

Using (3.5) in (3.4),

$$
\begin{aligned}
r< & \frac{M_{1} M_{4} q}{\Gamma(1+q)} \Omega\left(r^{\prime}+b H_{0}\left(1+r^{\prime}\right)\right)\left[1+\frac{M_{1} M_{2} M_{3} q}{\Gamma(1+q)} \sqrt{\frac{b^{2 q-1}}{2 q-1}}\right] \\
& +\frac{M_{1} M_{2} M_{3} q}{\Gamma(1+q)}\left[\left\|x_{1}\right\|+M_{1}\|\phi(0)\|\right] \sqrt{\frac{b^{2 q-1}}{2 q-1}} .
\end{aligned}
$$


Let $\xi=r^{\prime}+b H_{0}\left(1+r^{\prime}\right)$ and $c=\frac{M_{1} M_{4} q}{\Gamma(1+q)}$. Note that $\xi \rightarrow \infty$ as $r \rightarrow \infty$. Now dividing both sides of (3.6) by $r$ and taking the limit as $r \rightarrow \infty$, we get

$$
\begin{aligned}
1 \leq c & \lim _{\xi \rightarrow \infty} \inf \frac{\Omega(\xi)}{\xi} \cdot \frac{\xi}{r}\left[1+\frac{M_{1} M_{2} M_{3} q}{\Gamma(1+q)} \sqrt{\frac{b^{2 q-1}}{2 q-1}}\right] \\
& +\frac{1}{r} \cdot \frac{M_{1} M_{2} M_{3} q}{\Gamma(1+q)}\left[\left\|x_{1}\right\|+M_{1}\|\phi(0)\|\right] \sqrt{\frac{b^{2 q-1}}{2 q-1}} .
\end{aligned}
$$

Then, by $\left(\mathrm{H}_{2}\right)(\mathrm{ii})$, we get $1 \leq 0$. This is a contradiction. Hence, for some positive integer $r$, $\widetilde{\Phi}\left(B_{r}\right) \subseteq B_{r}$.

Step 2: $\widetilde{\Phi}$ is continuous on $B_{r}$.

For this purpose, we assume that $y^{(n)} \rightarrow y$ in $B_{r}$. Define

$$
\begin{aligned}
& F_{n}(s)=f\left(s, y_{s}^{(n)}+\hat{\phi}_{s}, \int_{0}^{s} h\left(s, \tau, y_{\tau}^{(n)}+\hat{\phi}_{\tau}\right) d \tau\right) \text { and } \\
& F(s)=f\left(s, y_{s}+\hat{\phi}_{s}, \int_{0}^{s} h\left(s, \tau, y_{\tau}+\hat{\phi}_{\tau}\right) d \tau\right) .
\end{aligned}
$$

Using $\left(\mathrm{H}_{2}\right)(\mathrm{i})$, (ii), $\left(\mathrm{H}_{3}\right)(\mathrm{i})$, (ii) and Lebesgue's dominated convergence theorem, we obtain

$$
\int_{0}^{t}(t-s)^{q-1}\left\|F_{n}(s)-F(s)\right\| d s \rightarrow 0 \quad \text { as } n \rightarrow \infty, t \in J .
$$

Now,

$$
\begin{aligned}
\left\|\widetilde{\Phi} y^{n}-\widetilde{\Phi} y\right\|_{\mathcal{C}} \leq & \frac{M_{1} q}{\Gamma(1+q)} \int_{0}^{t}(t-s)^{q-1}\left\|F_{n}(s)-F(s)\right\| d s \\
& +\sqrt{\frac{b^{2 q-1}}{2 q-1}} \frac{q M_{1} M_{2}}{\Gamma(1+q)}\left\|u_{y^{n}}-u_{y}\right\|_{L^{2}},
\end{aligned}
$$

where

$$
\left\|u_{y^{n}}-u_{y}\right\|_{L^{2}} \leq M_{3}\left[\int_{0}^{b}(b-s)^{q-1}\left\|F_{n}(s)-F(s)\right\| d s\right]
$$

Observing (3.7), (3.8) and (3.9), we have

$$
\left\|\widetilde{\Phi} y^{n}-\widetilde{\Phi} y\right\|_{\mathcal{C}} \rightarrow 0 \quad \text { as } n \rightarrow \infty
$$

which implies that $\widetilde{\Phi}$ is continuous on $B_{r}$.

Step 3: $\widetilde{\Phi}\left(B_{r}\right)$ is equicontinuous on $J$.

Indeed, let $z \in \widetilde{\Phi}\left(B_{r}\right)$ and $0 \leq t_{1}<t_{2} \leq b$. Then there is $y \in B_{r}$ such that

$$
\begin{aligned}
\| z\left(t_{2}\right) & -z\left(t_{1}\right) \| \\
\leq & \| \int_{0}^{t_{2}}\left(t_{2}-s\right)^{q-1} \mathcal{S}\left(t_{2}-s\right)\left[F(s)+B u_{y}(s)\right] d s \\
& \quad-\int_{0}^{t_{1}}\left(t_{1}-s\right)^{q-1} \mathcal{S}\left(t_{1}-s\right)\left[F(s)+B u_{y}(s)\right] d s \|
\end{aligned}
$$




$$
\begin{aligned}
\leq & \left\|\int_{t_{1}}^{t_{2}}\left(t_{2}-s\right)^{q-1} \mathcal{S}\left(t_{2}-s\right)\left[F(s)+B u_{y}(s)\right] d s\right\| \\
& +\left\|\int_{t_{1}-\epsilon}^{t_{1}}\left(t_{2}-s\right)^{q-1}\left[\mathcal{S}\left(t_{2}-s\right)-\mathcal{S}\left(t_{1}-s\right)\right]\left[F(s)+B u_{y}(s)\right] d s\right\| \\
& +\left\|\int_{t_{1}-\epsilon}^{t_{1}}\left[\left(t_{2}-s\right)^{q-1}-\left(t_{1}-s\right)^{q-1}\right] \mathcal{S}\left(t_{1}-s\right)\left[F(s)+B u_{y}(s)\right] d s\right\| \\
& +\left\|\int_{0}^{t_{1}-\epsilon}\left(t_{2}-s\right)^{q-1}\left[\mathcal{S}\left(t_{2}-s\right)-\mathcal{S}\left(t_{1}-s\right)\right]\left[F(s)+B u_{y}(s)\right] d s\right\| \\
& +\left\|\int_{0}^{t_{1}-\epsilon}\left[\left(t_{2}-s\right)^{q-1}-\left(t_{1}-s\right)^{q-1}\right] \mathcal{S}\left(t_{1}-s\right)\left[F(s)+B u_{y}(s)\right] d s\right\| .
\end{aligned}
$$

Using Lemma 2.2, we can verify that the right-hand side of the above inequality tends to zero as $t_{2} \rightarrow t_{1}$. Therefore, $\widetilde{\Phi}\left(B_{r}\right)$ is equicontinuous on $J$.

Step 4: The Mönch's condition holds.

Suppose that $W \subseteq B_{r}$ is countable and $W \subseteq \operatorname{conv}(\{0\} \cup \widetilde{\Phi}(W))$. We show that $\beta(W)=0$, where $\beta$ is the Hausdorff MNC. Without loss of generality, we may suppose that $W=$ $\left\{y^{n}\right\}_{n=1}^{\infty}$. Now we need to show that $\widetilde{\Phi}(W)(t)$ is relatively compact in $X$ for each $t \in J$.

By Theorem 2.1, we have

$$
\begin{aligned}
\beta_{V}\left(\left\{u_{y^{n}}(s)\right\}_{n=1}^{\infty}\right) \\
\leq K_{W}(s) \frac{2 M_{1} q}{\Gamma(1+q)} \int_{0}^{b}(b-s)^{q-1} \beta\left(F_{n}(s)\right) d s \\
\leq K_{W}(s) \frac{2 M_{1} q}{\Gamma(1+q)} \int_{0}^{b}(b-s)^{q-1} \eta(s)\left[\sup _{-\infty<\theta \leq 0} \beta\left(\left\{y^{n}(s+\theta)+\hat{\phi}(s+\theta)\right\}_{n=1}^{\infty}\right)\right. \\
\left.\quad+\beta\left(\left\{\int_{0}^{s} h\left(s, \tau, y_{\tau}^{n}+\hat{\phi}_{\tau}\right) d \tau\right\}_{n=1}^{\infty}\right)\right] d s \\
\leq K_{W}(s) \frac{2 M_{1} q}{\Gamma(1+q)} \int_{0}^{b}(b-s)^{q-1} \eta(s)\left(1+2 \zeta^{*}\right) \sup _{0 \leq \tau \leq s} \beta(W(\tau)) d s .
\end{aligned}
$$

Moreover, by Theorem 2.1 again

$$
\begin{aligned}
& \beta\left(\left\{\widetilde{\Phi}_{y^{n}}(s)\right\}_{n=1}^{\infty}\right) \\
& \leq \beta\left(\left\{\int_{0}^{t}(t-s)^{q-1} \mathcal{S}(t-s) F_{n}(s) d s\right\}_{n=1}^{\infty}\right) \\
&+\beta\left(\left\{\int_{0}^{t}(t-s)^{q-1} \mathcal{S}(t-s) B u_{y^{n}}(s) d s\right\}_{n=1}^{\infty}\right) \\
& \leq \frac{2 M_{1} q}{\Gamma(1+q)} \int_{0}^{t}(t-s)^{q-1} \eta(s) d s\left(1+2 \zeta^{*}\right) \sup _{0 \leq \tau \leq s} \beta(W(\tau)) \\
&+\left(1+2 \zeta^{*}\right) \frac{2 M_{1} M_{2} q}{\Gamma(1+q)}\left(\int_{0}^{t}(t-s)^{q-1} K_{W}(s) d s\right) \\
& \times\left[\frac{2 M_{1} q}{\Gamma(1+q)} \int_{0}^{b}(b-s)^{q-1} \eta(s) d s\right] \sup _{0 \leq \tau \leq s} \beta(W(\tau)) d s \\
& \leq {\left[\frac{2 M_{1} q}{\Gamma(1+q)}\left(1+2 \zeta^{*}\right) M_{6}+\frac{2 M_{1} M_{2} q}{\Gamma(1+q)} M_{5} \frac{2 M_{1} q}{\Gamma(1+q)}\left(1+2 \zeta^{*}\right) M_{6}\right] \sup _{0 \leq \tau \leq s} \beta(W(\tau)) d s . }
\end{aligned}
$$


That is,

$$
\beta(\widetilde{\Phi} W(t)) \leq\left[1+\frac{2 M_{1} M_{2} M_{5} q}{\Gamma(1+q)}\right]\left(1+2 \zeta^{*}\right) \frac{2 M_{1} M_{6} q}{\Gamma(1+q)} \sup _{0 \leq \tau \leq s} \beta(W(\tau)),
$$

which implies, by Lemma 2.3,

$$
\beta(\widetilde{\Phi}(W)) \leq L^{*} \beta(W)
$$

where $L^{*}$ is defined in condition (3.10). Thus, from Mönch's condition, we get

$$
\beta(W) \leq \beta(\operatorname{conv}(\{0\} \cup(\widetilde{\Phi}(W))))=\beta(\widetilde{\Phi}(W)) \leq L^{*} \beta(W),
$$

which implies that $\beta(W)=0$.

Hence, using Lemma 2.4, $\widetilde{\Phi}$ has a fixed point $y$ in $B_{r}$. Then $x=y+\hat{\phi}$ is a mild solution of system (1.1)-(1.2) satisfying $x(b)=x^{1}$. Therefore system (1.1)-(1.2) is controllable on $J$. This completes the proof.

\section{Example}

As an application, we consider the following fractional control system:

$$
\begin{aligned}
{ }^{C} D_{t}^{\frac{2}{3}} x(t, y)= & \frac{\partial^{2}}{\partial y^{2}} x(t, y)+W \mu(t, y) \\
& +\mu\left(t, \int_{-\infty}^{t} \mu_{1}(s-t) x(s, y) d s, \int_{0}^{t} \int_{-\infty}^{0} \mu_{2}(s, y, \tau-s) x(\tau, y) d \tau d s\right), \\
& q \in\left(\frac{1}{2}, 1\right), y \in[0, \pi], t \in J, \\
x(t, 0)=x(t, 1)=0, \quad t \geq 0, & x(t, y)=\psi(t, y), \quad t \in(-\infty, 0], y \in[0,1],
\end{aligned}
$$

where ${ }^{C} D_{t}^{\frac{2}{3}}$ is a Caputo fractional partial derivative, $\phi \in \mathcal{B}_{h}, \mu: J \times[0,1] \times[0,1]$ is continuous in $t$ and $\psi$ is continuous and satisfies certain smoothness conditions.

Let $X=Y=L^{2}(0,1)$ be endowed with the usual norm $\|\cdot\|_{L^{2}}$, and let $A: D(A) \subset X \rightarrow X$ be defined by $A w=w^{\prime \prime} ; w \in D(A)$, where $D(A)=\left\{w \in X: w^{\prime \prime} \in X, w(0)=w(1)=0\right\}$. It is well known that $A$ is an infinitesimal generator of a semigroup $\{T(t), t \geq 0\}$ in $X$ and is given by $T(t) w(s)=w(t+s)$ for $w \in X, T(t)$ is not a compact semigroup on $X$ with $\beta(T(t) D) \leq \beta(D)$, where $\beta$ is the Hausdorff MNC, and there exists $M_{1} \geq 1$ such that $\sup _{t \in J}\|T(t)\| \leq M_{1}$. Moreover, $t \rightarrow w\left(t^{\frac{2}{3}} \theta+s\right) x$ is equicontinuous [19] for $t \geq 0$ and $\theta \in(0, \infty)$.

Define

$$
\begin{aligned}
& { }^{C} D_{t}^{\frac{2}{3}} x(t)(y)=\frac{\partial^{\frac{2}{3}}}{\partial t^{\frac{2}{3}}} x(t, y), \\
& x(t)(y)=x(t, y), \\
& g(t, \phi)(y)=\int_{-\infty}^{t} \mu_{2}(t, y, s) \phi(s)(y) d s, \\
& f\left(t, \phi, \int_{0}^{t} g(s, \phi) d s\right)(y)=\mu\left(t, \int_{-\infty}^{0} \mu_{1}(s) \phi(s)(y) d s, \int_{0}^{t} g(s, \phi)(y) d s\right) .
\end{aligned}
$$


Let $B: X \rightarrow X$ be defined by

$$
(B u)(t)(y)=W \mu(t, y), \quad 0<y<1 .
$$

With this choice of $A, B$ and $f$, system (4.1) can be rewritten as

$$
\begin{aligned}
& { }^{C} D^{q} x(t)=A x(t)+f\left(t, x_{t}, \int_{0}^{t} h\left(t, s, x_{s}\right) d s\right)+B u(t), \quad q=\frac{2}{3} \in(0,1), t \in J, \\
& x_{0}=\phi \in \mathcal{B}_{h} .
\end{aligned}
$$

For $y \in(0,1)$, the linear operator $W$ is given by

$$
(W u)(y)=\int_{0}^{1}(1-s)^{\frac{-1}{3}} \mathcal{S}(1-s) w \mu(s, y) d s,
$$

where

$$
\begin{aligned}
& \mathcal{S}(t) w(s)=\frac{2}{3} \int_{0}^{\theta} \theta \xi_{\frac{2}{3}}(\theta) w\left(t^{\frac{2}{3}} \theta+s\right) d \theta, \\
& \xi_{\frac{2}{3}}(\theta)=\frac{3}{2} \theta^{\frac{-5}{2}} \bar{w}_{\frac{2}{3}}\left(\theta^{\frac{-3}{2}}\right), \\
& \bar{w}_{\frac{2}{3}}(\theta)=\frac{1}{\pi} \sum_{n=1}^{\infty}(-1)^{n-1} \theta^{\frac{-2 n+3}{3}} \frac{\Gamma\left(\frac{2 n+3}{3}\right)}{n !} \sin \left(\frac{2 n \pi}{3}\right), \quad \theta \in(0, \infty) .
\end{aligned}
$$

If we assume that $W$ satisfies $\left(\mathrm{H}_{4}\right)$, then all the conditions of Theorem 3.1 are satisfied. Hence, system (4.1)-(4.3) is controllable on $J$.

\section{Conclusions}

In this paper, abstract results concerning the controllability of fractional functional integro-differential systems with infinite delay involving the Caputo derivative in a Banach space are obtained. Upon making some appropriate assumptions, by utilizing the ideas and techniques of solution operators, the theory of fractional calculus and Mönch's fixed point theorem via measures of noncompactness, sufficient conditions for controllability results are obtained under some weakly compactness conditions. Finally, an example is presented in the end to show the applications of the obtained abstract results.

Competing interests

The authors declare that they have no competing interests.

\section{Authors' contributions}

The two authors contributed equally to the manuscript and typed, read and approved the final version.

\section{Author details}

${ }^{1}$ Department of Mathematics, RVS Faculty of Engineering, RVS Technical Campus, Coimbatore, Tamil Nadu 641402 , India. ${ }^{2}$ Department of Mathematics and Computer Science, Faculty of Arts and Sciences, Cankaya University, Ankara, 06530, Turkey. ${ }^{3}$ Department of Chemical and Materials Engineering, Faculty of Engineering, King Abdulaziz University, Jeddah, Saudi Arabia. ${ }^{4}$ Institute of Space Sciences, P.O. Box MG-23, Magurele-Bucharest, R 76900, Romania.

\section{Acknowledgements}

The authors are grateful to the referees for careful reading of the manuscript and giving some useful remarks. The first author would like to thank Dr. K.V. Kupusamy, Chairman and Dr. Y. Robinson, Director at RVS Technical Campus,

Coimbatore, Tamilnadu, India, for their constant encouragement and support during this research work. 


\section{References}

1. Kilbas, A, Srivastava, H, Trujillo, JJ: Theory and Applications of Fractional Differential Equations. Elsevier, Amsterdam (2006)

2. Lakshmikantham, V, Leela, S, Vasundhara Devi, J: Theory of Fractional Dynamic Systems. Cambridge Scientific Publishers, Cambridge (2009)

3. Miller, KS, Ross, B: An Introduction to the Fractional Calculus and Fractional Differential Equations. Wiley, New York (1993)

4. Podlubny, I: Fractional Differential Equations. Academic Press, New York (1999)

5. Baleanu, D, Diethelm, K, Scalas, E, Trujillo, JJ: Fractional Calculus Models and Numerical Methods. Series on Complexity, Nonlinearity and Chaos. World Scientific, Singapore (2012)

6. Tarasov, VE: Fractional Dynamics: Application of Fractional Calculus to Dynamics of Particles, Fields and Media. Springer, Heidelberg (2010)

7. Balachandran, K, Govindaraj, V, Rodriguez-Germa, L, Trujillo, JJ: Controllability results for nonlinear fractional order dynamical systems. J. Optim. Theory Appl. 156(1), 33-44 (2013)

8. Balachandran, K, Govindaraj, V, Rodriguez-Germa, L, Trujillo, JJ: Controllability of nonlinear higher order fractional dynamical systems. Nonlinear Dyn. 71(4), 605-612 (2013)

9. Wu, G, Baleanu, D: Variational iteration method for the Burgers' flow with fractional derivatives-new Lagrange multipliers. Appl. Math. Model. 37(9), 6183-6190 (2013)

10. dos Santos, JPC, Arjunan, MM, Cuevas, C: Existence results for fractional neutral integro-differential equations with state dependent delay. Comput. Math. Appl. 62(3), 1275-1283 (2011)

11. dos Santos, JPC, Vijayakumar, V, Murugesu, R: Existence of mild solutions for nonlocal Cauchy problem for fractional neutral integro-differential equation with unbounded delay. Commun. Math. Anal. 14(1), 59-71 (2013)

12. Hernández, E, O'Regan, D, Balachandran, K: On recent developments in the theory of abstract differential equations with fractional derivatives. Nonlinear Anal. 73, 3462-3471 (2010)

13. Hernández, E, O'Regan, D, Balachandran, K: Existence results for abstract fractional differential equations with nonlocal conditions via resolvent operators. Indag. Math. 24(1), 68-82 (2013)

14. Wang, JR, Zhou, Y: Existence and controllability results for fractional semilinear differential inclusions. Nonlinear Anal., Real World Appl. 12, 3642-3653 (2011)

15. Ravichandran, C, Baleanu, D: Existence results for fractional neutral functional integro-differential evolution equations with infinite delay in Banach spaces. Adv. Differ. Equ. 215, 1-12 (2013)

16. Klamka, J: Controllability of Dynamical Systems. Kluwer Academic, Dordrecht (1993)

17. Machado, JA, Ravichandran, C, Rivero, M, Trujillo, JJ: Controllability results for impulsive mixed-type functional integro-differential evolution equations with nonlocal conditions. Fixed Point Theory Appl. 66, 1-16 (2013)

18. Wang, JR, Zhou, Y: Complete controllability of fractional evolution systems. Commun. Nonlinear Sci. Numer. Simul. $17(11), 4346-4355(2012)$

19. Wang, JR, Fan, Z, Zhou, Y: Nonlocal controllability of semilinear dynamic systems with fractional derivative in Banach spaces. J. Optim. Theory Appl. 154(1), 292-302 (2012)

20. Ji, S, Li, G, Wang, M: Controllability of impulsive differential systems with nonlocal conditions. Appl. Math. Comput. 217, 6981-6989 (2011)

21. Debbouchea, A, Baleanu, D: Controllability of fractional evolution nonlocal impulsive quasilinear delay integro-differential systems. Comput. Math. Appl. 62(3), 1442-1450 (2011)

22. Feckan, M, Wang, JR, Zhou, Y: Controllability of fractional functional evolution equations of Sobolev type via characteristic solution operators. J. Optim. Theory Appl. 156(1), 79-95 (2013)

23. Ahmed, HM: Controllability for Sobolev type fractional integro-differential systems in a Banach space. Adv. Differ. Equ. $167,1-10(2012)$

24. Wang, F, Liu, Z: Complete controllability of fractional neutral differential systems in abstract space. Abstr. Appl. Anal. 2013, Article ID 529025 (2013). doi:10.1155/2013/529025

25. Yan, Z: Controllability of fractional-order partial neutral functional integrodifferential inclusions with infinite delay. J. Franklin Inst. 348, 2156-2173 (2011)

26. Vijayakumar, V, Ravichandran, C, Murugesu, R: Nonlocal controllability of mixed Volterra-Fredholm type fractional semilinear integro-differential inclusions in Banach spaces. Dyn. Contin. Discrete Impuls. Syst., Ser. B, Appl. Algorithms 20, 485-502 (2013)

27. Chang, Y-K: Controllability of impulsive functional differential systems with infinite delay in Banach spaces. Chaos Solitons Fractals 33, 1601-1609 (2007)

28. Yan, B: Boundary value problems on the half-line with impulses and infinite delay. J. Math. Anal. Appl. 259(1), 94-114 (2001)

29. Zhou, Y, Jiao, F: Existence of mild solutions for fractional neutral evolution equations. Comput. Math. Appl. 59 1063-1077 (2010)

30. Banas, J, Goebel, K: Measure of noncompactness in Banach spaces. In: Lecture Notes in Pure and Applied Mathematics. Dekker, New York (1980)

31. Obukhovski, $\vee$, Zecca, P: Controllability for systems governed by semilinear differential inclusions in a Banach space with a noncompact semigroup. Nonlinear Anal. 70, 3424-3436 (2009)

32. O'Regan, D, Precup, R: Existence criteria for integral equations in Banach spaces. J. Inequal. Appl. 6, 77-97 (2001)

33. Mönch, $\mathrm{H}$ : Boundary value problems for nonlinear ordinary differential equations of second order in Banach spaces. Nonlinear Anal. 4, 985-999 (1980)

34. Quinn, MD, Carmichael, N: An approach to nonlinear control problems using fixed point methods, degree theory and pseudo-inverses. Numer. Funct. Anal. Optim. 7, 197-219 (1984-1985) 